\title{
Indicadores de regulação autonômica cardíaca em repouso e durante exercício progressivo. Aplicação do limiar de variabilidade da freqüência cardíaca
}

\author{
Lenise Fronchetti $1,2,3$ \\ Fábio Nakamura ${ }^{2,3}$ \\ César Aguiar ${ }^{2}$ \\ Fernando Oliveira ${ }^{1}$
}

https://doi.org/10.5628/rpcd.06.01.21

\author{
${ }_{1}^{1}$ Universidade Estadual de Santa Catarina \\ Laboratório de Pesquisa Morfo-Funcional \\ Brasil \\ ${ }^{2}$ Universidade Estadual de Londrina \\ Centro de Educação Física e Desportos \\ Brasil \\ ${ }_{3}$ Universidade Estadual de Londrina \\ Grupo de Estudo das Adaptações Fisiológicas ao \\ Treinamento \\ Brasil
}

\begin{abstract}
Indexes of Autonomic Cardiac Regulation in Rest and During Progressive Exercise. Application of the Heart Rate Variability Threshold.

This study aimed to verify the degree of association between the resting heart rate $\left(H R_{\text {Rest }}\right)$, different resting heart rate variability indexes and the exercise intensity related to the heart rate threshold variability. Twenty men $(21,3 \pm 2,6$ years old $)$ began the test protocol with a resting period sitting on a cycle ergometer and then were submitted to a progressive test $(14,6 \mathrm{~W} /$ minute) until exhaustion. In the first load, the threshold variability was identified as lower than $3 \mathrm{~ms}$ in the decreasing variability HR curve. The HR and the variability were registered with a Polar ${ }^{\circledR}$ heart rate device. Spearman Rank $(r)$ correlation was used to calculate the associations among these variables $(p<0,05)$. The correlations between various variability indexes were statistically significant with $r \geq 0,80$. The $H R_{\text {Rest }}$ showed a significant and a negative association with the variability indexes and with the threshold variability intensity $(r \geq-0,63)$. On the other hand, the threshold variability intensity showed a close relationship with the following indexes: SD1 $(r=0,51)$, SD2 $(r=0,46)$, RMSSD $(r=0,48), p N N 50(r=$ $0,55), H F(r=0,50)$ e LF/HF $(r=-0,56)$. These results showed that an elevate resting vagal activity can postpone the increase of the predominance of the sympathetic system during progressive exercises.
\end{abstract}

entre a frequiência cardíaca de repouso $\left(\mathrm{FC}_{\mathrm{Rep}}\right)$, diferentes índices de variabilidade da freqüência cardíaca (FC) em repouso e a intensidade de esforço referente ao limiar de variabilidade da FC. Vinte homens $(21,3 \pm 2,6$ anos $)$ iniciaram o protocolo do teste com um período de repouso sentado no cicloergômetro, em seguida realizaram um teste progressivo (14,6 W/minuto) até a exaustão. $\mathrm{O}$ limiar de variabilidade foi identificado na primeira carga inferior a $3 \mathrm{~ms}$ na curva de decréscimo da variabilidade da FC. A FC e sua variabilidade foram registradas utilizando-se um cardiofreqüencímetro Polar ${ }^{\circledR}$. Para associação dessas variáveis (Spearman Rank) foi considerado $\mathrm{p}<0,05$. As correlações entre os diversos índices de variabilidade foram signifi-

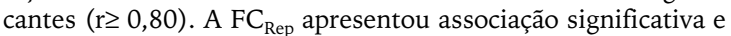
negativa com os índices de variabilidade e com a intensidade no limiar de variabilidade da FC $(r \geq-0,63)$. A intensidade no limiar mostrou estreita relação com os índices: SD1 $(r=0,51)$, SD2 $(r=0,46)$, RMSSD $(r=0,48)$, pNN50 $(r=0,55)$, HF $(r$ $=0,50)$ e LF/HF $(r=-0,56)$. Nossos achados indicam que elevada atividade vagal de repouso pode postergar o aumento da predominância simpática em exercício progressivo.

Palavras-chave: freqüência cardíaca de repouso, variabilidade da freqüência cardíaca, domínio do tempo e freqüência, limiar de variabilidade da freqüência cardíaca, capacidade aeróbia.
Key Words: rest heart rate, heart rate variability, time and frequency domain, heart rate variability threshold, aerobic capacity. 


\section{INTRODUÇÃO}

Os valores de freqüência cardíaca em repouso $\left(\mathrm{FC}_{\mathrm{Rep}}\right)$ são comumente utilizados como referência de condição funcional do organismo, influenciando inclusive na determinação de faixas de intensidade de exercício, de acordo com alguns modelos vigentes (15). Em geral, baixos valores de $\mathrm{FC}_{\text {Rep }}$ refletem uma boa condição funcional, enquanto que altos valores estariam aparentemente relacionados com distúrbios fisiológicos e predisposição para a ocorrência de doenças cardiovasculares $(13,17,22)$.

Por sua vez, a variabilidade da freqüência cardíaca (VFC) é um parâmetro de avaliação da funcionalidade neurocardíaca, já que a modulação autonômica, por meio dos ramos simpáticos e parassimpáticos que agem sobre o coração, influencia de forma direta e diferencial as oscilações nessa variável (28). A mensuração da VFC, por várias técnicas, também permite diagnosticar indivíduos com riscos para enfermidades cardiovasculares. Assim, diversos estudos têm utilizado a análise da VFC, por meio da quantificação das flutuações dos intervalos R-R, como meio não invasivo de estimar o tônus simpático e parassimpático sobre o nodo sinoatrial, sob diversas condições fisiológicas, sobretudo no exercício agudo e em diversas fases de treinamento, incluindo estágios de overtraining $(2,10,12,24,29)$. A magnitude das flutuações da FC pode ser indicadora de disfunção autonômica cardíaca (3), sendo que a redução na $\mathrm{FC}$ em repouso $\left(\mathrm{FC}_{\mathrm{Rep}}\right)$ e o aumento da atividade vagal estão normalmente associados a uma melhora no nível de aptidão física relacionada à saúde $(1,9)$. Sugere-se que a estimulação vagal apresenta um efeito protetor sobre a vulnerabilidade elétrica ventricular, ao contrário, uma baixa atividade parassimpática estaria correlacionada ao desenvolvimento de arritmias letais (26). Desse modo, a análise do perfil autonômico cardíaco representa um elemento importante para a estratificação de risco de prevalência de algumas doenças cardíacas.

Normalmente, dois métodos são utilizados para quantificação da VFC: um refere-se ao domínio de tempo, o qual emprega ou índices extraídos diretamente das variações temporais dos intervalos $\mathrm{R}-\mathrm{R}$ em milissegundos (SD, RMSSD, SD1, SD2), ou percentuais de medidas absolutas de intervalos R-R acumulados acima de um valor de referência
(pNN50). O outro tipo de análise, no domínio da freqüência, define e separa, por análise espectral, as diferentes intensidades de sinais a diferentes freqüências, observadas nas variações do sinal eletrocardiográfico (HF e LF) (Quadro 1) $(11,25,28)$. Lima e Kiss (18), utilizando o índice SD1 da plotagem de Poincaré, apresentaram a possibilidade de identificação de um limiar de VFC (LiVFC), correspondente à carga associada ao valor inferior a $3 \mathrm{~ms}$ na curva de decréscimo da VFC em função da intensidade em teste incremental (Figura 1). Nesse mesmo estudo, os autores compararam o LiVFC com o limiar de lactato e verificaram que ambos são identificados em cargas similares de esforço $(r=$ $0,76)$. Resultados semelhantes foram encontrados por Bruneto et al. (8), comparando e correlacionando o LiVFC com o limiar ventilatório $(r=0,66)$. Assim, o LiVFC estaria associado à transição entre intensidade de esforço com predominante influência vagal no controle da FC, para intensidades sob predominância simpática $(18,31)$.

Dessa forma, o LiVFC pode ser considerado um indicador da capacidade aeróbia e, assim, ser utilizado como parâmetro fisiológico para prescrição de exercício e treinamento físico $(18,21)$.

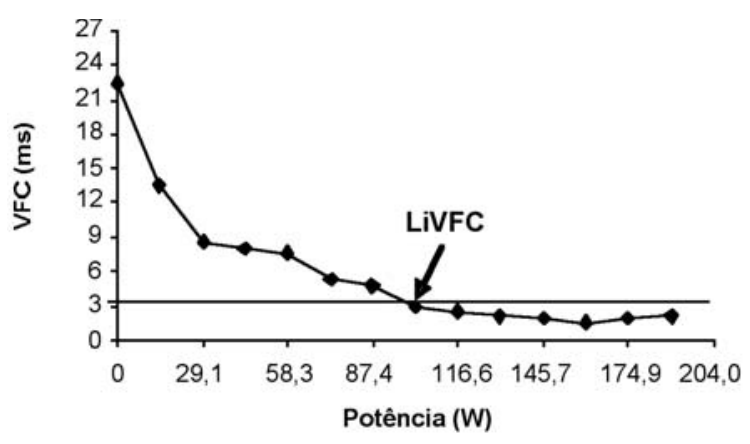

Figura 1 - VFC durante exercício progressivo e identificação do LiVFC.

Considerando que o estado do balanço entre as atividades simpática e parassimpática sobre o coração reveste-se de importante significado funcional, e que a $\mathrm{FC}_{\text {Rep }}$, os índices de VFC de repouso e a intensidade no LiVFC são indicadores da modulação autonômica, o objetivo do presente estudo foi verificar o 
grau de associação entre essas variáveis. A hipótese era a de que diferentes índices de VFC de repouso poderiam predizer a retirada vagal em exercício, demarcada a partir do LiVFC.

\section{METODOLOGIA}

\section{Amostra}

Vinte homens jovens $(21,3 \pm 2,6$ anos; $72,9 \pm 10,2$ $\mathrm{kg}$; e 178,5 $\pm 6,0 \mathrm{~cm}$ ), aparentemente saudáveis, não-atletas e não praticantes de exercícios físicos foram convidados a participar do estudo, com consentimento informado obtido conforme a Declaração de Helsinki (4).

\section{Material}

Foi utilizado um cicloergômetro de frenagem mecânica Monark ${ }^{\circledR}$ e um cardiofreqüencímetro Polar ${ }^{\circledR}$ modelo S810i que permite o registro da VFC (23, 30). A análise da VFC e o cálculo da FC média de cada estágio, foram realizados por meio do software Polar Precision Performance ${ }^{\circledR}$.

\section{Protocolo do teste incremental}

Os indivíduos foram instruídos a não ingerirem bebidas alcoólicas e produtos com cafeína, além de não praticarem exercícios físicos nas $24 \mathrm{~h}$ antecedentes ao teste.

Ao chegarem no local do teste, os participantes passaram por uma anamnese, na qual havia algumas perguntas sobre o estado de saúde e informações pessoais, com o intuito de detectar possíveis contraindicações à participação no estudo. Após esse procedimento-padrão, os sujeitos foram posicionados no cicloergomêtro e permaneceram por três minutos em repouso, acomodados sentados sobre o assento do cicloergômetro, a fim de a FC alcançar valores estáveis próximos aos de repouso absoluto. Em seguida, iniciava-se o teste de esforço progressivo máximo em cicloergômetro sem carga, com incrementos de $14,6 \mathrm{~W}$ a cada minuto (60 rpm), até à exaustão voluntária ou à incapacidade de manutenção da rotação requerida (18).

A partir dos dados de FC, obtidos batimento-a-batimento, foi possível determinar a FC, em bpm, e a VFC de repouso, por meio dos seguintes índices de análise no domínio do tempo: SD, SD1, SD2, RMSSD e pNN50; e no domínio da freqüência: LF,
HF e LF/HF (ver definições no Quadro 1), assim como identificar o LiVFC, que correspondeu à primeira carga onde a média dos intervalos R-R, expressa por meio do índice SD1 da plotagem de Poincaré (30), foi inferior a 3 (ms) (18).

\begin{tabular}{|c|c|c|}
\hline Índice & Unidade & Definição \\
\hline SD & $\mathrm{ms}$ & Desvio padrão de todos os intervalos R-R. \\
\hline RMSSD & ms & $\begin{array}{l}\text { Raiz quadrada da média das diferenças } \\
\text { sucessivas ao quadrado, entre R-R adja- } \\
\text { centes. }\end{array}$ \\
\hline pNN50 & $\%$ & $\begin{array}{l}\text { Percentagem das diferenças sucessivas } \\
\text { entre os intervalos R-R que são > } 50 \text { ms. }\end{array}$ \\
\hline SD1 & $\mathrm{ms}$ & $\begin{array}{l}\text { Desvio padrão dos intervalos R-R instantâ- } \\
\text { neos. }\end{array}$ \\
\hline SD2 & ms & $\begin{array}{l}\text { Desvio padrão dos intervalos R-R analisa- } \\
\text { dos em longo prazo. }\end{array}$ \\
\hline HF & $\mathrm{Hz}$ ou $\mathrm{ms}^{2}$ & $\begin{array}{l}\text { Componente espectral de alta freqüência } \\
(0,15-0,4 \mathrm{~Hz}]\end{array}$ \\
\hline LF & $\mathrm{Hz}$ ou $\mathrm{ms}^{2}$ & $\begin{array}{l}\text { Componente espectral de baixa freqüência } \\
(0,04-0,15 \mathrm{~Hz}) \text {. }\end{array}$ \\
\hline LF/HF & $\%$ & Relação entre os componentes LF e HF \\
\hline
\end{tabular}

Fontes: Grupi [11], Lima e Kiss (18].

\section{Tratamento estatístico}

$\mathrm{Na}$ análise de distribuição dos dados foi mostrado que algumas variáveis estudadas não apresentavam distribuição normal, identificada através do teste Shapiro-Wilk (5). Portanto, foi aplicado o teste não paramétrico de Spearman Rank para correlacionar a $\mathrm{FC}_{\text {Rep }}$, os índices de VFC e a intensidade no LiVFC (PLiVFC) $(p<0,05)$. Os tratamentos foram processados no software SPSS ${ }^{\circledR} 11.0$.

\section{RESULTADOS}

A estatística descritiva das variáveis analisadas neste estudo está apresentada na tabela 1 . 
Tabela 1: Valores médios e desvio-padrão das variáveis avaliadas durante o repouso e o exercício.
Valores*

Repouso
$\mathrm{FC}_{\text {Rep }}(\mathrm{bpm})$
$\mathrm{SD}(\mathrm{ms})$
$\mathrm{SD} 1(\mathrm{~ms})$
$\mathrm{SD} 2$ (ms)
$\mathrm{RMSSD}(\mathrm{ms})$
pNN50 (\%)
$\mathrm{LF}\left(\mathrm{ms}^{2}\right)$
$\mathrm{HF}\left(\mathrm{ms}^{2}\right)$
LF/HF $(\%)$
Exercício
PLiVFC (W)
Potência de Pico (W)
Potência Relativa $[\%]$
FC no LIVFC (bpm)

$130 \pm 9$
Tabela 3: Coeficiente de correlação entre os parâmetros de VFC no domínio do tempo e da freqüência com a freqüência cardíaca de repouso, a freqüência cardíaca no LiVFC e a potência no LiVFC.

\begin{tabular}{lccc} 
& $\mathrm{FC}_{\text {Rep }}$ & FCLiVFC & PLiVFC \\
\hline SD & $-0,78^{*}$ & 0,15 & 0,42 \\
SD1 & $-0,89^{*}$ & 0,12 & $0,51^{*}$ \\
SD2 & $-0,81^{*}$ & 0,15 & $0,46^{*}$ \\
RMSSD & $-0,84^{*}$ & 0,19 & $0,48^{*}$ \\
pNN50 & $-0,84^{*}$ & 0,20 & $0,55^{*}$ \\
LF & $-0,71^{*}$ & 0,02 & 0,41 \\
HF & $-0,81^{*}$ & 0,31 & $0,50^{*}$ \\
LF/HF & $0,76^{*}$ & $-0,37$ & $-0,56^{*}$ \\
FC & - & $-0,45$ & $-0,63^{*}$ \\
FCLiVFC & - & - & 0,34
\end{tabular}

*Grau de associação significante entre as variáveis $(p<0,05)$.

$109,3 \pm 29,3$

$227,3 \pm 30,0$

$48,3 \pm 11,3$

A $\mathrm{FC}_{\mathrm{Rep}}$ apresentou correlação significativa e inversa tanto com os índices de VFC no domínio do tempo,

Nas tabelas 2 e 3 estão expressas as variáveis correlacionadas pelo teste de Spearman Rank. Verificou-se que, de forma geral, os índices que expressam a VFC no domínio do tempo apresentaram correlações significantes entre si e com os componentes da análise espectral (HF, LF e LF/HF), da mesma forma estes últimos apresentaram significante grau de associação entre si.

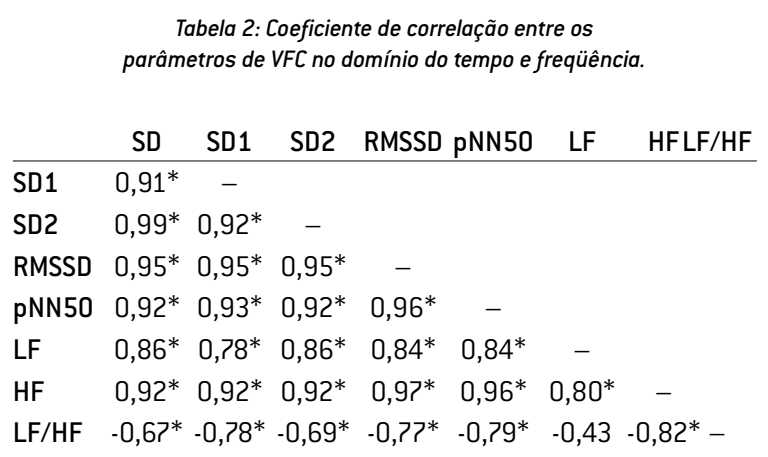

*Grau de associação significante entre as variáveis $(p<0,05)$. quanto com os índices no domínio da freqüência, e, ainda, com a PLiVFC. Entretanto, com o LF/HF a relação foi positiva. Além disso, observou-se que a intensidade de esforço no LiVFC apresentou valores de correlação moderados, mas significantes (exceto $\mathrm{SD}$ e LF), com os diferentes índices de VFC. A figura 2 ilustra essas correlações.

a

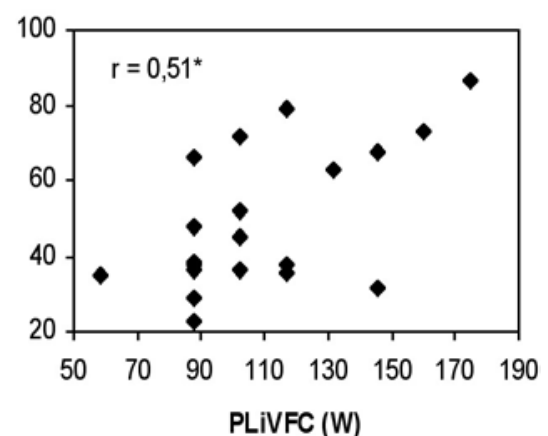

b

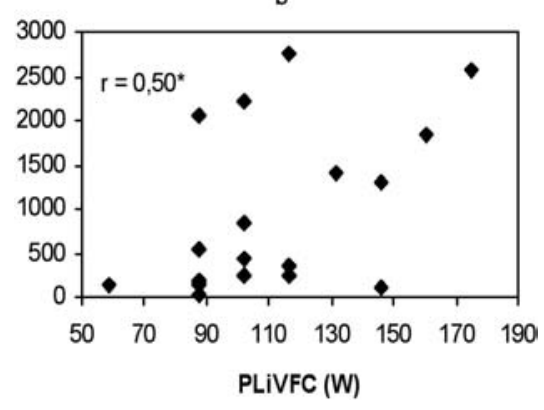


C

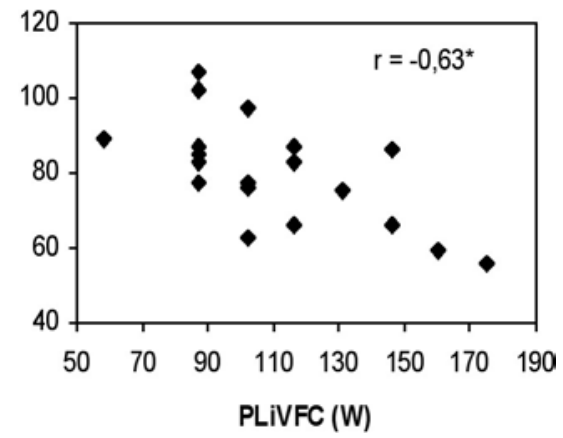

d

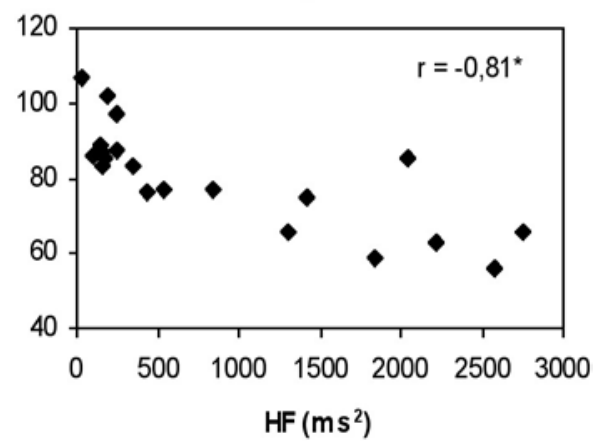

Figura 2: Gráficos de dispersão representativos da relação entre - a: $S D 1$ vs PLiVFC; b: HF vs PLiVFC; c: FC Rep vs PLiVFC; d: FC Rep vs HF. O valor de " $r$ " corresponde ao coeficiente de correlação de Spearman $\left({ }^{*} p<0,05\right]$.

\section{DISCUSSÃO}

As medidas no domínio do tempo e da freqüência expressam o mesmo fenômeno de variação de batimentos cardíacos adjacentes, sob diferentes tratamentos formais dos dados experimentais. Assim, algumas associações entre os índices que traduzem a VFC têm sido demonstradas $(6,25,30)$. Contudo, este estudo descreve, pela primeira vez, associações dessas variáveis no indivíduo em repouso com um indicador de limiar de transição fisiológica durante o exercício.

A literatura reporta que os índices SD, SDANN (desvio-padrão da média dos intervalos $\mathrm{R}-\mathrm{R}$, medida em segmentos de 5 minutos) e SDNNi (média dos desvios-padrão dos intervalos R-R, medida em segmentos de 5 minutos) traduzem a variabilidade global e refletem a atividade parassimpática e simpática. Ao passo que o pNN50 e o RMSSD, por considerarem diferenças entre os intervalos R-R adjacentes, quantificam variações rápidas da $\mathrm{FC}$ e, consequentemente, refletem predominância do tônus vagal $(16,25,28)$. Dessa forma, os achados do presente estudo corroboram os da literatura $(6,25,30)$, ao verificar que o SD apresenta forte associação com os índices de VFC no domínio do tempo (SD1: $r=0,91$; SD2: $\mathrm{r}=$ 0,99; RMSSD: $r=0,95$ ) e da freqüência (LF: $r=$ 0,86; HF: $r=0,92$; LF/HF: $r=-0,67)$. No entanto, o SD não apresenta correlação significativa com a PLiVFC. Vale ressaltar que o LiVFC pode demarcar um período de transição da retirada vagal para uma predominância simpática no controle da FC.

Portanto, parece que os índices que expressam ação com predominância de um componente de modulação autonômica - parassimpático (pNN50, RMSSD, SD1 e HF) - apresentam uma maior correlação com a intensidade alcançada no LiVFC $(r=0,55 ; r=$ 0,$48 ; r=0,51$ e $r=0,50$, respectivamente). Desse modo, pode-se inferir que indivíduos que apresentam elevados valores dos índices pNN50, RMSSD, SD1 e HF em condição de repouso tendem a alcançar o LiVFC em intensidades de exercício maiores, refletindo numa ação mais prolongada da atividade parassimpática durante o exercício progressivo e, por conseguinte, tendem a apresentar melhor aptidão aeróbia.

Esses achados são, em parte, confirmados por Mourot et al. (20). Segundo os autores, atletas de endurance que apresentavam sintomas clínicos de overtraining manifestaram menores valores em repouso na posição supino de HF e SD1, e maiores valores de LF/HF do que os treinados sem overtraining, assemelhando-se aos indivíduos controle sedentários. A dispersão dos dados individuais na plotagem de Poincaré permitiu a discriminação dos sujeitos nas diferentes condições de treinamento, sendo os pontos mais dispersos no estado treinado do que em overtraining e na situação controle. Ou seja, os indicadores de menor tônus parassimpático em repouso predispõem os indivíduos a menores níveis de desempenho físico.

No presente estudo, a PLiVFC $(109,3 \pm 29,3 \mathrm{~W})$ foi identificada em potência similar ao reportado por Lima e Kiss (18) $(110,5 \pm 18,5$. W), e ligeiramente superior à encontrada por Nakamura et al. (21) $(89,1$ $\pm 28.7 \mathrm{~W}$ ), em amostras semelhantes. Os percentuais 
relativos à carga máxima de ocorrência do LiVFC encontrados nestes estudos se assemelham aos dos estudos citados ( $48 \%, 49 \%$ e $46 \%$, respectivamente). Trabalhos recentes têm abordado as adaptações crônicas da regulação autonômica cardíaca ao treinamento. Nakamura et al. (21), investigaram, em cicloergômetro, as modificações no LiVFC após três semanas de treinamento aeróbio, verificando que a VFC (analisada por meio do índice SD1), tanto em repouso quanto em cargas de trabalho submáximo, parece sofrer alterações em resposta ao treinamento físico, apontando para um maior tônus vagal. Logo, as adaptações autonômicas decorrentes dessa intervenção propiciaram uma melhora na VFC de repouso (de $37 \pm 13 \mathrm{~ms}$ para $46 \pm 17 \mathrm{~ms}$ ), na $\mathrm{FC}_{\text {Rep }}$ (de $89 \pm 13 \mathrm{bpm}$ para $83 \pm 9 \mathrm{bpm})$ e na intensidade de esforço associada ao LiVFC (pré-treinamento: 89,1 $\pm 28,7 \mathrm{~W} / 46 \%$; pós-treinamento: $123,1 \pm 32,9 \mathrm{~W}$ / 60\%; em valores absolutos e relativos, respectivamente). Desse modo, esses resultados contribuíram para evidenciar elementos a favor da validade do LiVFC como indicador de capacidade aeróbia. Nossos achados parecem reforçar este enunciado, ao verificar grau de associação significante entre diferentes índices de VFC de repouso e a PLiVFC, sugerindo que a magnitude da atividade vagal cardíaca de repouso estaria relacionada a uma retirada vagal tardia durante o exercício físico, sendo o LiVFC identificado em maior intensidade de esforço.

Nossos resultados sugerem, também, que uma baixa $\mathrm{FC}_{\text {Rep }}$ se relaciona com elevada VFC de repouso $(\mathrm{r} \geq$ $-0,71)$, do mesmo modo que corresponde a uma maior PLiVFC $(r=-0,63)$, indicando, novamente, que a alta atividade vagal em repouso resulta em um efeito prolongado do tônus parassimpático durante a realização do exercício físico progressivo. Por outro lado, verificou-se que os valores de $\mathrm{FC}_{\mathrm{Rep}}$ foram superiores aos comumente encontrados na literatura, devido, provavelmente, às diferenças metodológicas empregadas. Em geral, uma medida representativa da $\mathrm{FC}_{\text {Rep }}$ é obtida durante o período de sono ou logo após acordar (14), sendo que indivíduos saudáveis e não atletas, avaliados na posição supino, apresentam valores de $\mathrm{FC}_{\mathrm{Rep}}$ numa faixa de 65 a $75 \mathrm{bpm}$ $(7,19)$. Entretanto, outros estudos mostram valores em torno de $75 \mathrm{bpm}$, quando esta variável foi men- surada na posição sentado $(2,27)$. Yamamoto et al. (32) também utilizaram a metodologia de medida da $\mathrm{FC}_{\mathrm{Rep}}$ com os indivíduos sentados no cicloergômetro. No entanto, estes permaneceram 20 minutos em repouso, o que pode ter proporcionado uma diminuição mais acentuada da $\mathrm{FC}_{\text {Rep }}(68 \pm 3 \mathrm{bpm})$. Outros estudos mostram que a $\mathrm{FC}_{\text {Rep }}$ e a FC submáxima são modificadas em resposta ao treinamento físico $(15,21,29)$, sendo que a redução pode ser creditada tanto às adaptações na regulação intrínseca de despolarização do miocárdio, quanto às adaptações na modulação autonômica cardíaca (32). Desse modo, nossos resultados corroboram os da literatura e verificam, pela primeira vez, a associação entre diversos índices de VFC e a FC em repouso e o LiVFC, sustentando o modelo tradicionalmente aceito.

Neste contexto, Yamamoto et al. (32), durante um programa de treinamento aeróbio, verificaram, inicialmente, uma concomitante redução da $\mathrm{FC}_{\text {Rep }} \mathrm{e}$ aumento dos índices de modulação parassimpática. Porém, a partir do $28^{\circ}$ dia de treinamento, a redução da $\mathrm{FC}_{\text {Rep }}$ ocorreu sem o aumento da VFC. Esses achados confirmam a associação inversa entre essas variáveis encontrada no presente estudo, no entanto, sugerem que as modificações autonômicas contribuem, parcialmente, para a diminuição da $\mathrm{FC}_{\text {Rep }}$. Esta dissociação parece estar na dependência da continuação das alterações cardíacas, como o volume e diâmetro diastólico ventricular esquerdo e estabilização das modificações na regulação autonómica, após o primeiro mês de treinamento (42 dias).

Em síntese, nossos resultados evidenciam que indivíduos que apresentam, simultaneamente, valores baixos de $\mathrm{FC}_{\text {Rep }}$ e elevados de VFC de repouso, tendem a alcançar o LiVFC em intensidades de esforço maior, indicando que uma alta atividade vagal de repouso, além de sugerir uma boa condição da função cardiovascular, parece também estar relacionada à capacidade aeróbia. Além disso, os índices SD1, SD2, RMSSD, pNN50, HF e LF/HF em repouso, apresentam significante associação com a variável indicadora de aptidão aeróbia, representada pelo LiVFC. Desse modo, parece que a partir das variáveis de repouso e exercício que foram analisadas, é possível fazer inferências quanto à regulação autonômica cardíaca e à capacidade aeróbia dos sujeitos. 


\section{REFERÊNCIAS}

1. Almeida MB, Araújo CGS (2003). Effects of aerobic training on heart rate. Rev Bra Med Esp 9 (2): 104 - 112.

2. Alonso DO, Forjaz CLM, Rezende LO, Braga AMFW, Barreto ACP, Negrão CE, Randon MUPB (1998). Comportamento da freqüência cardíaca e da sua variabilidade durante as diferentes fases do exercício físico progressivo máximo. Arq Bras Cardiol 71(6): 787 - 792.

3. Appel ML, Berger RD, Saul JP, Smith JM, Cohen RJ (1989). Beat to beat variability in cardiovascular variables: noise or music? J Am Coll Cardiol 14: 1139 - 1148.

4. Associação Médica Mundial: Declaração de Helsinki V. 1996. Disponível em: < http:/ www.bioetica.ufrgs.br/helsin5.htm>. Acesso em 15 abril 2005.

5. Barros MVG, Reis RS (2003). Análise de dados em atividade física e saúde. Londrina: Midiograf.

6. Brennan M, Palaniswami M, Kamen P (2002). Poincaré plot interpretation using a physiological model of HRV based on a network of oscillators. Am J Physiol Heart Circ Physiol 283: H1873 - H1886.

7. Brooks GA, Fahey TO (1984). Exercise Physiology: Human Bionergetics and its Aplications. New York: John Wiley and Sons.

8. Brunetto BC, Nakamura FY, Hirai DM, Roseguini BT, Brunetto AF (2004). Comparação do limiar de variabilidade de freqüência cardíaca com o limiar ventilatório em indivíduo adultos saudáveis. In XXVII Simpósio Internacional de Ciências do Esporte. Edição Especial da Revista Brasileira de Ciência e Movimento. São Caetano do Sul: Celafiscs, 40.

9. Dixon EM, Kamath MV, McCartney N, Fallen EL (1992). Neural regulation of heart rate variability in endurance athletes and sedentary controls. Cardiovasc Res 26: 713 719.

10. Gall B, Parkhouse W, Goodman D (2004). Heart rate variability of recently concussed athletes at rest and exercise. Med Sci Sports Exerc 36(8):1269 - 1274.

11. Grupi CJ (1998). Variabilidade da Freqüência Cardíaca. Jornal Diagnósticos \& Cardiologia. 1. ed. fev./mar./abr. 1998. Disponível em: <http:/www.cardios.com.br/jornal02/tese.htm>. Acesso em: 21 março 2005.

12. Hautala A (2004). Effect of physical exercise on autonomic regulation of heart rate. Academic Dissertation (Faculty of Medicine) - University of Oulu, Finland.

13. Jeukendrup A, Van Diemen A (1998). Heart rate monitoring during training and competition in cyclists. J Sports Sci 16: S91 - S99.

14. Jeukendrup A, Hesselink MKC, Snyder AC, Kuipers H, Keiser HA (1992). Physiological changes in male competitive cyclists after two weeks of intensified training. Int $J$ Sports Med 13: 534 - 541

15. Karvonen MJ, Kentala E, Mustala O (1957). The effects of training on heart rate: a longitudinal study. Ann Med Exp Biol Fenn 35 (3):307 - 315.

\section{CORRESPONDÊNCIA}

\section{Lenise Fronchetti}

Desembargador Sálvio Gonzaga, 126/402

88080-020 - Coqueiros

Florianópolis - SC

BRASIL

lefronchetti@yahoo.com.br
16. Kautzner J, Hnatkova K (1995). Correspondence of differents methods for heart rate variability measurement. In Malik M, Camm AJ (ed.) Heart Rate Variability. New York: Futura, $119-126$.

17. Kenney WL (1985). Parasympathetic control of resting heart rate: relationship to aerobic power. Med Sci Sports Exerc 17: $451-455$.

18. Lima JRP, Kiss MAPDA (1999). Limiar de variabilidade da freqüência cardíaca. Rev Bras Ativ Fis Saúde 4 (1): 29 - 38. 
19. Melanson EL (2000). Resting heart rate variability in men varying in habitual physical activity. Med Sci Sports Exerc 32 (11): $1894-1901$.

20. Mourot L, Bouhaddi M, Perrey S, Cappelle S, Henriet MT, Wolf JP, Rouillon JD, Regnard J (2004). Decrease in heart rate variability with overtraining: assessment by the Poincaré plot analysis. Clin Physiol Funct Imaging 24: 10 18.

21. Nakamura FY, Aguiar CA, Fronchetti L, Aguiar AF, Perrout de Lima JR (No prelo). Alteração do limiar de variabilidade da frequiência cardíaca após treinamento aeróbio de curto prazo. Motriz (UNESP).

22. Palatini $P$ (1999). Need for a revision of the normal limits of resting heart rate. J Hypertens 33: $622-625$.

23. Peltola K, Hannula M, Held T, Kinnunen H, Nissilä S, Laukkanen R, Marti B (2000). Validity of polar fitness test based on heart rate variability in assessing $\mathrm{VO}_{2}$ max in trained individuals. (Abstract). In Proc. 5th Annual Congress of ECSS. Jyväskylä, Finland, 565.

24. Pichot V, Busso T, Roche F, Garet M, Costes F, Duverney D, Lacour JR, Barthe'Le'My JC (2002). Autonomic adaptations to intensive and overload training periods: a laboratory study. Med Sci Sports Exerc 34 (10): 1660 - 1666.

25. Rassi A Jr (2000). Compreendendo melhor as medidas de análise da variabilidade da freqüência cardíaca. Jornal Diagnósticos \& Cardiologia. 20. ed., abr/mai/jun. 2000. Disponível em: <http: /www.cardios.com.br/jornal20/métodos\%20diagnosticos.htm>. Acesso em: 17 março 2005.

26. Reis AF, Bastos BG, Mesquita BT, Romêo $F^{\circ}$ LJM, Nóbrega ACL (1998). Disfunção parassimpática, variabilidade da frequência cardíaca e estimulação colinérgica após infarto agudo do miocárdio. Arq Bras Cardiol 70(3): 193 - 199.

27. Roecker K, Niess AM, Horstmann T, Striegel H, Mayer F, Dickhuth HH (2002). Heart rate prescriptions from performance and anthropometrical characteristics. Med Sci Sports Exerc 34 (5): $881-887$.

28. Task Force of the European Society of Cardiology and the North American Society of Pacing and Electrophysiology: Heart rate variability (1996). Standards of measurement, physiological interpretation, and clinical use. Circulation 93: 1043 - 1065.

29. Tulppo MP, Hautala AJ, Mäkikallio TH, Laukkanen RT, Nissilä S, Hughson RL, Huikuri HV (2003). Effects of aerobic training on heart rate dynamics in sedentary subjects. J Appl Physiol 95: $364-372$.

30. Tulppo MP, Mäkikallio TH, Takala T, Seppänen T, Huikuri H (1996). Quantitative Beat-To-Beat Analysis Of Heart Rate Dynamics During Exercise. Am J Physiol 271: H244 252.

31. Tulppo MP, Mäkikallio TH, Seppänen T, Laukkanen RT, Huikuri HV (1998). Vagal modulation of heart rate during exercise: effects of age and physical fitness. American Journal of Physiology (Heart Circ. Physiol.) 274(2): H424H429.

32. Yamamoto K, Miyachi M, Saitoh T, Yoshioka A, Onodera S (2001). Effects of endurance training on resting and postexercise cardiac autonomic control. Med Sci Sports Exerc 33 (9): $1496-1502$. 